\title{
A Boiler-Turbine System Control Using A Fuzzy Auto-Regressive Moving Average (FARMA) Model
}

\author{
Un-Chul Moon and Kwang Y. Lee, Fellow, IEEE
}

\begin{abstract}
This paper presents an application of an online self-organizing fuzzy logic controller to a boiler-turbine system of fossil power plant. The control rules and the membership functions of the proposed fuzzy logic controller are generated automatically without using a plant model. A boiler-turbine system is described as a multi-input multioutput (MIMO) nonlinear system in this paper. Then, three single-loop fuzzy logic controllers are designed independently. Simulation shows robust results for various kinds of electric load demand changes and parameter variations of boiler-turbine system.
\end{abstract}

Index Terms-Boilers, fuzzy control, self-organizing control.

\section{INTRODUCTION}

A boiler-turbine system supplies high-pressure steam to rotate the generator in thermal electric power generation. The purpose of the boiler-turbine system control is to meet the load demand of electric power while maintaining the pressure and water level in the drum within tolerance. To design a controller, the boiler-turbine system is usually modeled as a multiinput multioutput (MIMO) nonlinear system [1].

The severe nonlinearity and wide operation range to the boiler-turbine plant have resulted in many challenges of power system control engineers. Hogg and Ei-Rabaie presented an application of self-tuning generalized predictive control (GPC) to a boiler system [2]. Rovnak and Corlis presented an application of dynamic matrix control to fossil power plant [3]. Though [2] and [3] both optimize the performance on a receding horizon, the self-tuning GPC uses an online identification model, while the dynamic matrix control uses offline step-response model of the plant. Ben-Abdennour and Lee applied robust control method for a power plant [4]. They decomposed a power system to boiler, turbine, and generator, and applied the linear quadratic Gaussian with loop transfer recovery (LQG/LTR) to two subsystems, boiler, and turbine, as local controllers.

To overcome the nonlinearity of the boiler-turbine plant, many kinds of artificial intelligence techniques have also been applied. Prasad, Swidenbank, and Hogg proposed a predictive ' control based on an NN model [5]. They used offline training of neural network to capture the nonlinearity of power plant dynamics. And the performance of receding horizon is minimized by real-time optimization. Dimeo and Lee used a genetic

Manuscript received May 22, 2002.

This work was supported in part by the Information Telecommunication Research Institute of Chung-Ang University.

U.-C. Moon is with the School of Electrical Engineering, Chung-Ang University, Seoul 156-756, Korea (e-mail: ucmoon@cau.ac.kr).

K. Y. Lee is with the Department of Electrical Engineering, The Pennsylvania State University, University Park, PA 16802 USA (e-mail: kwanglee@psu.edu). Digital Object Identifier 10.1109/TEC.2002.808408 algorithm (GA) to enhance the wide range performance of PI controller or linear quadratic regulator (LQR) [6]. In that paper, the parameters of conventional PI controller or LQR are found by GA for wide operation range of boiler-turbine system. Alturki and Abdennour applied a neural-fuzzy control to a boiler-turbine system [7]. They trained neuro-fuzzy system with the data from five LQRs which are designed for each operating point. Cheung and Wang presented a comparison of fuzzy and PI controller for drum-boiler system [8] and concluded that the fuzzy control system has better performance than PID control system especially in setpoint tracking.

In this paper, a self-organizing fuzzy logic controller (SOFLC) proposed in [9], called fuzzy auto-regressive moving average (FARMA) controller, is applied to the boiler-turbine system. In [9], we proposed a complete design method for an online SOFLC without using the mathematical model. The FARMA fuzzy logic controller (FLC) was successfully applied to several kinds of power system stabilizer design [10], [11]. In contrast to a conventional FLC, where the rule base and membership functions are supplied by an expert or tuned offline through experiment, the FARMA FLC needs no experts in making control rules. Instead, rules are generated using the history of input-output data. The generated rules are stored in the fuzzy rule space and updated online by a self-organizing procedure.

The single-loop control scheme is applied to control the boiler-turbine system in this paper. That is, three input-output pairs of boiler-turbine system are determined. Then, three FARMA FLCs are applied independently to the three single loops. Simulation considers various kinds of electric load demand changes and parameter variations of boiler-turbine system.

\section{FARMA FUZZY LOGIC CONTROLLER}

\section{A. FARMA Rule}

The self-organizing FARMA fuzzy logic controller is reviewed briefly [9]. A single-input single-output (SISO) system can be described with a function or a mapping of the input-output history.

$$
y(k+1)=f(y(k), y(k-1), \ldots, u(k), u(k-1), \ldots)
$$

where $y(k)$ and $u(k)$ are, respectively, the output and input variables at the $k$-th time step.

The objective of the control problem is to find a control input sequence which will drive the system to an arbitrary reference set point $y_{\text {ref }}$. Rearranging (1) for control purposes, the value of 
the input $u$ at the $k$-th step that is required to yield the reference output $y_{r e f}$ can be written as follows:

$$
u(k)=g\left(y_{r e f}, y(k), y(k-1), \ldots, u(k-1), u(k-2), \ldots\right)
$$

which is viewed as an inverse mapping of (1) for the setpoint.

The system (1) yields the last output value $y(k+1)$ when the output and input values $y(k), y(k-1), y(k-2), \ldots, u(k), u(k-$ $1), u(k-2), \ldots$ are given. This implies that $u(k)$ is the input to be applied when the desired output is $y_{\text {ref }}$ as indicated explicitly in (2). Therefore, a FARMA rule with the input and output history is defined as follows:

$$
\begin{aligned}
& \text { IF } y_{\text {ref }} \text { is } A_{1 i}, y(k) \text { is } A_{2 i}, \\
& y(k-1) \text { is } A_{3 i}, \ldots, y(k-n+1) \text { is } A_{(n+1) i}, \\
& A N D u(k-1) \text { is } B_{1 i}, \\
& u(k-2) \text { is } B_{2 i}, \ldots, u(k-m) \text { is } B_{m i}, \\
& \text { THEN } u(k) \text { is } C_{i}, \text { (for the } i \text {-th rule) }
\end{aligned}
$$

where

$n, m \quad$ number of output and input variables;

$A_{i j}, B_{i j}$ antecedent linguistic values for the $i$-th rule;

$C_{i} \quad$ consequence linguistic value for the $i$-th rule.

Unlike a conventional FLC, where an expert gives the linguistic values $A_{i j}, B_{i j}$, and $C_{i}$, and makes rules, these linguistic values are determined from the crisp values of the input and output history at each sampling time. Therefore, the assigned $u(k)$ may not be a good control initially. However, the rule base is updated each time using the self-organizing procedure and better controls are applied as time progresses.

The linguistic values $A_{i j}, B_{i j}$, and $C_{i}$ are obtained by fuzzifying the corresponding crisp values of $y$ and $u$. The fuzzification is done for a crisp value on a reasonably assumed input or output range. When an assumed input or output range is $[a, b]$, the membership function for a crisp value $x_{1}$ is defined in a triangular shape as follows:

$$
\mu_{A_{1}}=\left\{\begin{array}{cl}
1+\frac{\left(x-x_{1}\right)}{(b-a)}, & \text { if } a \leq x<x_{1}, \\
1-\frac{\left(x-x_{1}\right)}{(b-a)}, & \text { if } x_{1} \leq x<b, \\
0 & \text { else }
\end{array}\right.
$$

The above fuzzification procedure generates a FARMA rule at each sampling step and stores in a rule base. This means that every experience is regarded initially as a fuzzy logic control rule. As the run continues, the knowledge will be accumulated and the FARMA rule is updated in the rule space.

\section{B. Inference and Defuzzification}

When a new set of input and output data is sampled, its "truth value" is determined with respect to each rule and the net linguistic control action $C_{n}$ is deduced with the $\varphi$-operation [12] as follows:

$$
\begin{aligned}
C_{n} & =\cap_{i}\left(\omega_{i} \varphi \mu_{C_{i}}\right) \\
\omega_{i} \varphi \mu_{C_{i}} & = \begin{cases}1 & \text { if } \omega_{i} \leq \mu_{C_{i}} \\
\mu_{C_{i}} & \text { if } \omega_{i}>\mu_{C_{i}}\end{cases}
\end{aligned}
$$

where

$C_{n} \quad$ net linguistic control action;

$\omega_{i} \quad$ truth value of the $i$-th rule;

$\mu_{C i}$ membership degree of the consequence linguistic value $C_{i}$ in the $i$-th rule.

By taking the $\alpha$-cut of the $C_{n}$ where $\alpha=\max \mu\left(C_{n}\right)$, the net control range (NCR) is determined as the subset $[p, q]$ of $[a, b]$ with the constant membership value $\alpha$ as the highest possibility.

Defuzzification is performed to determine a crisp value from the NCR resulting from the inference. First, the NCR is modified by using a prediction or "trend" of the output response. For example, the series of the last outputs can be extrapolated in time domain to estimate $y(k+1)$ using the Newton backward-difference formula.

Defuzzification is performed by comparing the two values, the estimate $\hat{y}(k+1)$ and the reference output $y_{\text {ref }}$, or the temporary target $y_{r}(k+1)$ generated by

$$
y_{r}(k+1)=y(k)+\beta\left(y_{r e f}-y(k)\right)
$$

where $\beta$ is the target ratio constant $(0<\beta \leq 1)$. The value of $\beta$ describes the rate with which the present output $y(k)$ approaches the reference output value, and thus, has a positive value between 0 and 1 . The value of $\beta$ is chosen by the user to obtain a desirable response.

When the estimate exceeds the reference or the target output, the control has to slow down. Otherwise, the control should speed up. To modify the control range, the sign of $\Delta u(k)(=$ $u(k)-u(k-1))$ is assumed to be the same as the sign of $\left(y_{r}(k+1)-\hat{y}(k+1)\right)$ without the loss of generality. Thus, when $y_{r}(k+1)>\hat{y}(k+1)$, hence the sign of $\Delta u(k)$ is positive, $u(k)$ has to be increased from the previous input $u(k-1)$. On the other hand, when the sign of $\Delta u(k)$ is negative, $u(k)$ has to be decreased from the previous input $u(k-1)$. This limits the range of control and modifies the NCR. The final crisp control value $u(k)$ is then selected as one of the midpoints of the modified NCR as follows:

$$
u(k)= \begin{cases}\frac{(u(k-1)+q)}{2}, & \text { for } y_{r}(k+1)>\hat{y}(k+1) \\ \frac{(p+u(k-1))}{2}, & \text { for } y_{r}(k+1)<\hat{y}(k+1)\end{cases}
$$

where $p$ and $q$ are the respective lower and upper limits of the NCR.

\section{Self-Organization of the Rule Base}

The FARMA rule defined in Section II-A is generated at every sampling time. Each rule can be represented as a point in the $(n+m+1)$-dimensional rule space [i.e., $\left.\left(x_{1 i}, x_{2 i}, \ldots, x_{(n+m+1) i}\right)\right]$. To update the rule base, the following performance index is defined

$$
J=\left|y_{r}(k+1)-y(k+1)\right|
$$

where $y(k+1)$ is the real plant output and $y_{r}(k+1)$ is the reference output. Therefore, at the $(k+1)$-th step, the performance index $J$ is calculated with the real plant output $y(k+1)$, resulting from the $k$-th step control. 


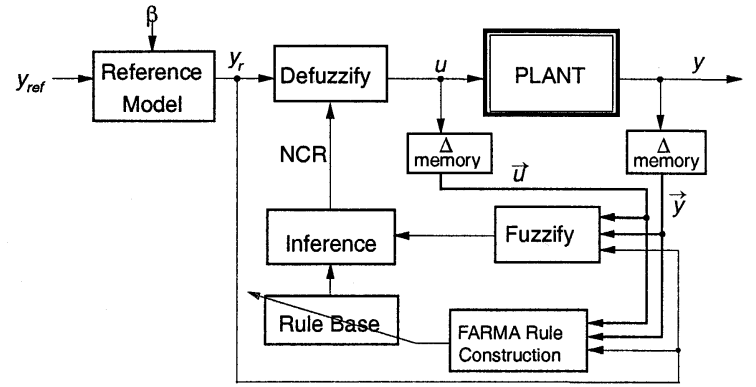

Fig. 1. FARMA control system architecture.

The fuzzy rule space is partitioned into a finite number of domains and only one rule (i.e., a point), is stored in each domain. If there is a new rule in domain with the smaller value of $J$, the old rule is replaced by the new one. The self-organization of the rule base, in other words "learning" of the object system, is performed at each sampling time as shown in Fig. 1.

\section{BOILER-TURBINE SYSTEM CONTROL}

\section{A. Boiler-Turbine System Model}

The model of Bell and Åström [1] is used in the simulation for the nonlinear boiler-turbine system. It was developed for a 160-MW oil-fired drum-type boiler-turbine-generator system for overall wide-range simulation. The model is a third-order MIMO nonlinear system described as follows [1]:

$$
\begin{aligned}
& \dot{x}_{1}=-0.0018 u_{2} x_{1}^{9 / 8}+0.9 u_{1}-0.15 u_{3} \\
& \dot{x}_{2}=\frac{\left[\left(0.73 u_{2}-0.16\right) x_{1}^{9 / 8}-x_{2}\right]}{10} \\
& \dot{x}_{3}=\frac{\left[141 u_{3}-\left(1.1 u_{2}-0.19\right) x_{1}\right]}{85} \\
& y_{1}=x_{1} \\
& y_{2}=x_{2} \\
& y_{3}=0.05\left(0.13073 x_{3}+100 a_{c s}+\frac{q_{e}}{9-67.975}\right)
\end{aligned}
$$

where

$$
\begin{aligned}
\alpha_{c s}= & \frac{\left(1-0.001538 x_{3}\right)\left(0.8 x_{1}-25.6\right)}{x_{3}\left(1.0394-0.0012304 x_{1}\right)} \\
q_{e}= & \left(0.854 u_{2}-0.147\right) x_{1} \\
& +45.59 u_{1}-2.514 u_{3}-2.096 .
\end{aligned}
$$

The three state variables $x_{1}, x_{2}$, and $x_{3}$ are drum steam pressure $\left(P\right.$ in $\left.\mathrm{kg} / \mathrm{cm}^{2}\right)$, electric power ( $E$ in MW), and steam-water fluid density in the drum $\left(\rho_{f}\right.$ in $\left.\mathrm{kg} / \mathrm{m}^{2}\right)$, respectively. The three outputs $y_{1}, y_{2}$, and $y_{3}$ are drum steam pressure $\left(x_{1}\right)$, electric power $\left(x_{2}\right)$, and drum water level deviation ( $L$ in $\mathrm{m}$ ), respectively. The $y_{3}$, drum water level $L$, is calculated using two algebraic calculations $\alpha_{c s}$ and $q_{e}$ which are the steam quality (mass ratio) and the evaporation rate $(\mathrm{kg} / \mathrm{s})$, respectively.

The three inputs $u_{1}, u_{2}$, and $u_{3}$ are normalized positions of valve actuators that control the mass flow rates of fuel, steam to the turbine, and feedwater to the drum, respectively. Positions of

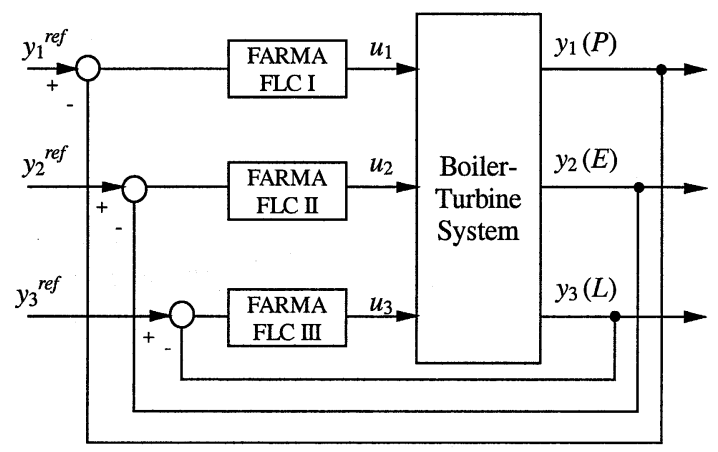

Fig. 2. Boiler-Turbine control system.

valve actuators are constrained to [0.1] and their rates of change per second are limited to

$$
\begin{aligned}
-0.007 & \leq \frac{d u_{1}}{d t} \leq 0.007 \\
-2.0 & \leq \frac{d u_{2}}{d t} \leq 0.02 \\
-0.05 & \leq \frac{d u_{3}}{d t} \leq 0.05 .
\end{aligned}
$$

\section{B. FARMA FLC Application to Boiler-Turbine System}

In this paper, the FRAMA FLC for SISO system (1) is directly applied to the boiler-turbine system. From the input-output point of view, the boiler-turbine system (10)-(20) is a three-input and three-output system. Therefore, three single loops, which are three FARMA FLCs, are applied independently in this paper.

The dominant input-output fairs should be determined first to design the single-loop configuration. From (10), the $x_{1}$ can be controlled by $u_{1}$ and $u_{2}$. Considering that the order of $x_{1}$ is about 100 in normal operation, $u_{2}$ term is smaller than $u_{1}$ term, which means $y_{1}$ (pressure) is dominantly affected by $u_{1}$ (mass flow rates of fuel). Therefore, the first FARMA FLC loop is to control $y_{1}$ with $u_{1}$. And from (11), $x_{2}$ is affected by $u_{2}$. Therefore, the second FARMA FLC is to control $y_{2}$ (electric power) with $u_{2}$ (steam to the turbine). Finally, from the physical property of boiler-turbine system, $y_{3}$ (drum water level deviation) is controlled by $u_{3}$ (feedwater to the drum). Therefore, the third FARMA FLC is to control $y_{3}$ with $u_{3}$.

The overall structure to control the boiler-turbine system is shown in Fig. 2. In Fig. 2, FARMA FLC I, II, and III control $y_{1}$ (pressure), $y_{2}$ (electric power), and $y_{3}$ (drum water level deviation) with $u_{1}$ (mass flow rates of fuel), $u_{2}$ (steam to the turbine) and $u_{3}$ (feedwater to the drum), respectively.

The orders $n$ and $m$ in (3) are 3 and 1 for each FRAMA FLC. Therefore, (3) for each FRAMA FLC is as follows:

$$
\begin{aligned}
& \text { IF } y_{\text {ref }} \text { is } A_{1 i}, y(k) \text { is } A_{2 i}, \\
& \quad y(k-1) \text { is } A_{3 i}, A N D u(k-1) \text { is } B_{1 i} \\
& \text { THEN } u(k) \text { is } C_{i} \quad \text { (for the } i \text {-th rule). }
\end{aligned}
$$

The output ranges for (4) are [70 150] $\left(P\right.$ in kilograms $\left./ \mathrm{cm}^{2}\right)$, [10 190] ( $E$ in megawatts) and [ $-0.50 .5]$ ( $L$ in meters) for FARMA FLC I, II, and III, respectively. The input ranges are [0 1] for each FARMA FLC. 


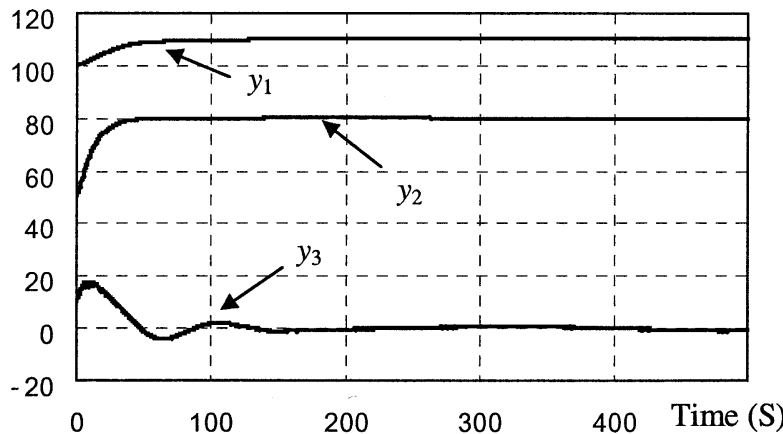

Fig. 3. Outputs of case 1.

\section{Simulation Results}

The control system and process model were developed with C-language in a personal-computer environment. Sampling times for simulations are $0.5 \mathrm{~s}$. Simulations presented here are to evaluate the control system performance for various kinds of electric load demand changes and parameter variations of boiler-turbine system and characteristics of single-loop controls with three FARMA FLCs. In [4], they pointed out that the pressure setpoint is increased through a functional mapping as the electric load is increased. Therefore, the pressure setpoints are also increased as electric loads increased in simulations.

With the assumption that the system is in a steady state with $X=(100,50,449.5), Y=(100,50,0)$, $U=(0.271,0.604,0.336)$ initially, we consider the seven cases to validate the proposed control system. The first four cases are to consider various kinds of electric load demands and the second three cases are to consider the parameter variations.

\section{A. Electric Load Demand Changes}

In this case study, four cases with various kinds of electric load demand changes are considered as follows: .

Case 1) $y_{1}^{\text {ref }}=110, y_{2}^{\text {ref }}=80, Y_{3}^{\text {ref }}=0$

Case 2) $y_{1}^{\text {ref }}=120, y_{2}^{\text {ref }}=100, y_{3}^{\text {ref }}=0$

Case 3) $y_{1}^{\text {ref }}=130, y_{2}^{\text {ref }}=120, y_{3}^{\text {ref }}=0$

Case 4) $\begin{cases}y_{1}^{\text {ref }}=110, y_{2}^{\text {ref }}=80, y_{3}^{\text {ref }}=0, & 0 \leq t \leq 400 \\ y_{1}^{\text {ref }}=120, y_{2}^{\text {ref }}=100, y_{3}^{\text {ref }}=0, & 400 \leq t \leq 800 \\ y_{1}^{\text {ref }}=130, y_{2}^{\text {ref }}=120, y_{3}^{\text {ref }}=0, & 800 \leq t \leq 1200 .\end{cases}$

Case 1 describes that the setpoints of pressure and electric load demand are increased to 110 and 80 , respectively, while the drum water level is kept to zero. Cases 2 and 3 describe the cases that the setpoints of pressure and electric load demand are increased to larger values for wider-range operation. Case 4 is to demonstrate the learning ability of the FARMA FLC. The setpoints of pressure and electric load demand are increased to the values of Cases 1, 2, and 3, but successively in every $400 \mathrm{~s}$ while the drum water level is kept to zero. Therefore, new plant experience is added to a rule base as new setpoints are applied.

Figs. 3-10 show the simulation results for cases 1, 2, 3, and 4. In the plots, units for output variables are $\left(\mathrm{kg} / \mathrm{cm}^{2}\right)$ for $y_{1}$, (MW) for $y_{2}$ and $(\mathrm{cm})$ for $y_{3}$ and units for input variables are normalized positions of valve actuators for three inputs $u_{1}, u_{2}$, and $u_{3}$.

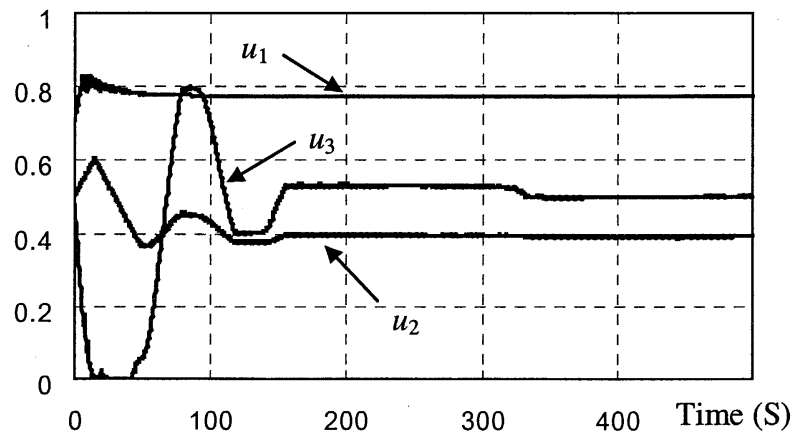

Fig. 4. Inputs of case 1.

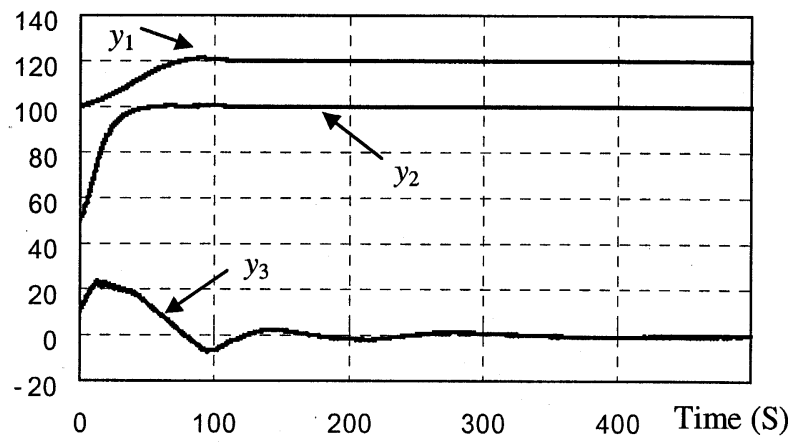

Fig. 5. Outputs of case 2 .

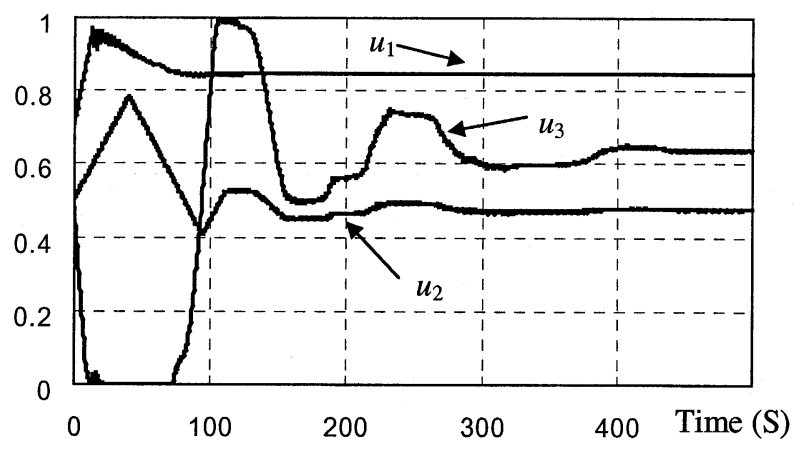

Fig. 6. Inputs of case 2

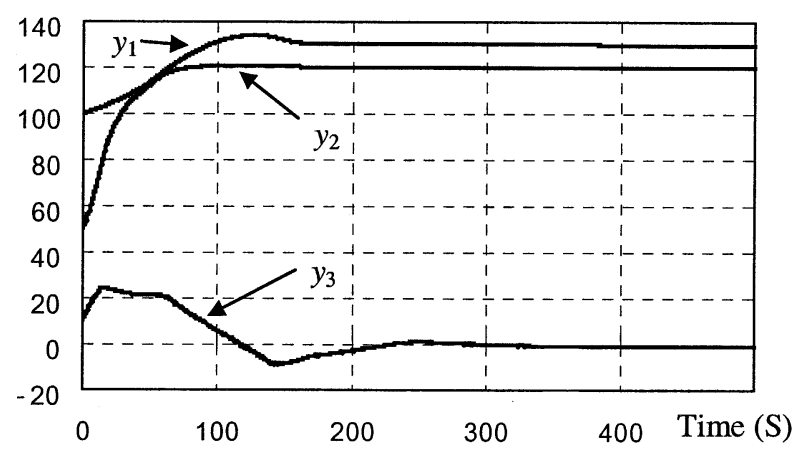

Fig. 7. Outputs of case 3 .

Fig. 3 shows the outputs for case 1 . The output $y_{1}$ tracks the reference 110 after $60 \mathrm{~s}$ and the $y_{2}$ is reached to the reference 80 in about $50 \mathrm{~s}$. The drum water level is initially increased to 20 , but returns to zero after $60 \mathrm{~s}$. Fig. 4 shows the control actions of case 1. For larger step increases (cases 2 and 3), the outputs respond similarly, but take longer to reach the setpoint and with 


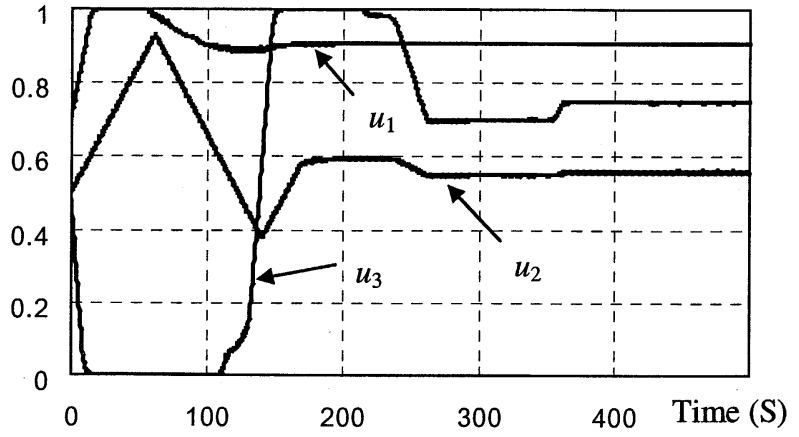

Fig. 8. Inputs of case 3.

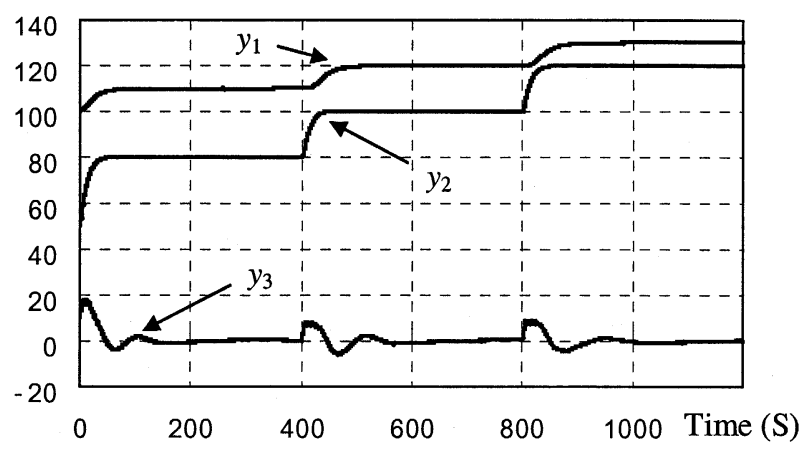

Fig. 9. Outputs of case 4 .

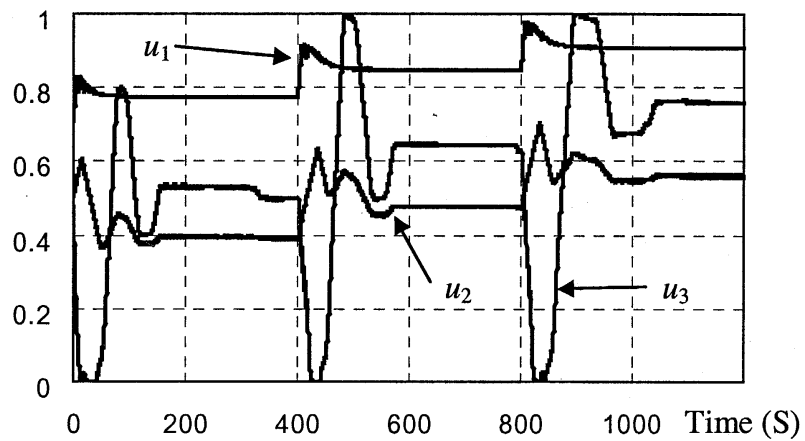

Fig. 10. Inputs of case 4.

more overshoots (Figs. 5 and 7). However, input signals are getting larger and often saturated as in Figs. 6 and 8. Figs. 9 and 10 show the outputs and inputs for case 4, which is to demonstrate the learning ability of FARMA FLC. The outputs $y_{1}$ and $y_{2}$ track their references in every $400 \mathrm{~s}$. The drum water level deviation is 20 at the first reference change, but reduced to 10 at the subsequent changes Fig. 9. On the contrary, in cases 2 and 3, the drum water level deviation is 20 and takes longer to settle down (Figs. 5 and ). In case 4, however, the drum water level deviations is 10 for the same level of setpoints as cases 2 and 3 (Fig. 9). This is because there is no rule at the initial setpoint change, but the experience on the first setpoint change was accumulated in the rule space and was used for the second setpoint change. Similarly, the experience gained with the first and the second setpoint changes were accumulated and used for the third setpoint change. As the controller experiences new inputs, the knowledge base is increased and the performance of the FARMA FLC improves.

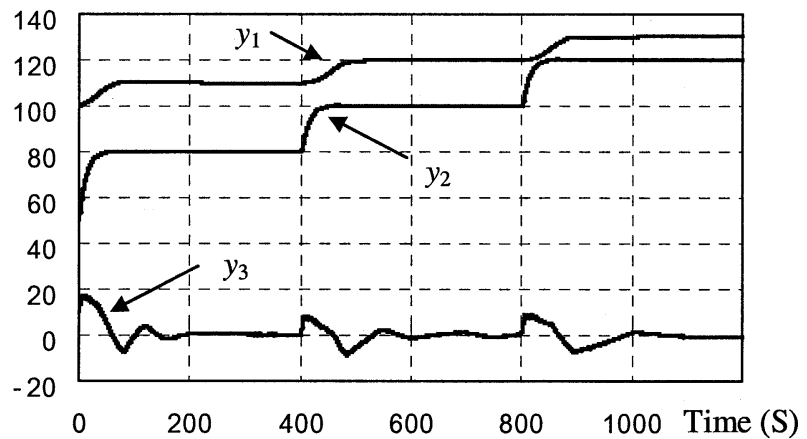

Fig. 11. Outputs of case 5.

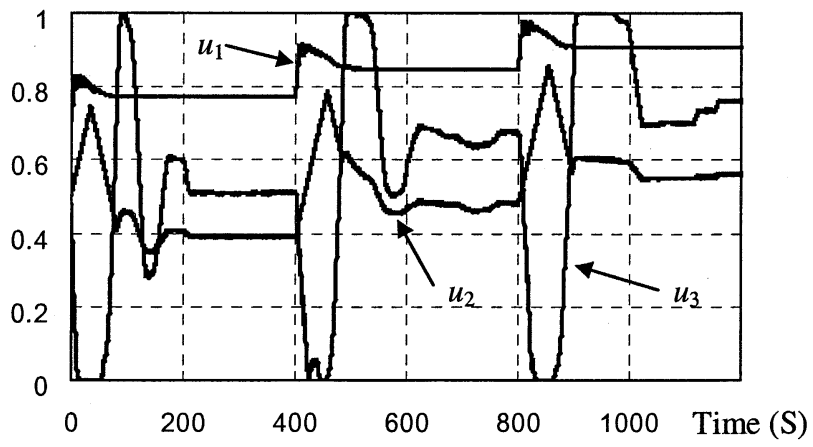

Fig. 12. Inputs of case 5 .

\section{B. Parameter Variations}

Three different cases for parameter variations are considered as follows:

Case 5) $50 \%$ change in model parameters in (10);

Case 6) 50\% change in model parameters in (10), (11), and (12);

Case 7) $50 \%$ change in the steam quality (mass ratio) $\alpha_{c s}$ in (15).

In case 5, three coefficients in (10) are reduced to $50 \%$ as follows:

$$
\dot{x}_{1}=-0.0009 u_{2} x_{1}^{9 / 8}+0.45 u_{1}-0.075 u_{3} .
$$

In case 6 , all coefficients in the three state equations are reduced to $50 \%$ [i.e., in addition to (22)]

$$
\begin{aligned}
& \dot{x}_{2}=\frac{\left[\left(0.365 u_{2}-0.08\right) x_{1}^{9 / 8}-0.5 x_{2}\right]}{10} \\
& \dot{x}_{3}=\frac{\left[70.5 u_{3}-\left(0.55 u_{2}-0.095\right) x_{1}\right]}{170} .
\end{aligned}
$$

In case 7, the coupling constant 100 in (15) is reduced to $50 \%$ as follows:

$$
y_{3}=0.05\left(0.13073 x_{3}+50 a_{c s}+\frac{q_{e}}{9}-67.975\right) .
$$

The setpoint changes of outputs for cases 5, 6, and 7 are the same as those in case 4. Figs. 11-16 show the simulation results for cases 5, 6, and 7 .

Compared to the base case, case 4, the output responses of cases 5 and 6 are closed to each other and the base case Figs. 11 and 13. However, control efforts are much larger (Figs. 12 and 14). Figs. 15 and 16 are for case 7. In Fig. 15, $y_{3}$ is better than 


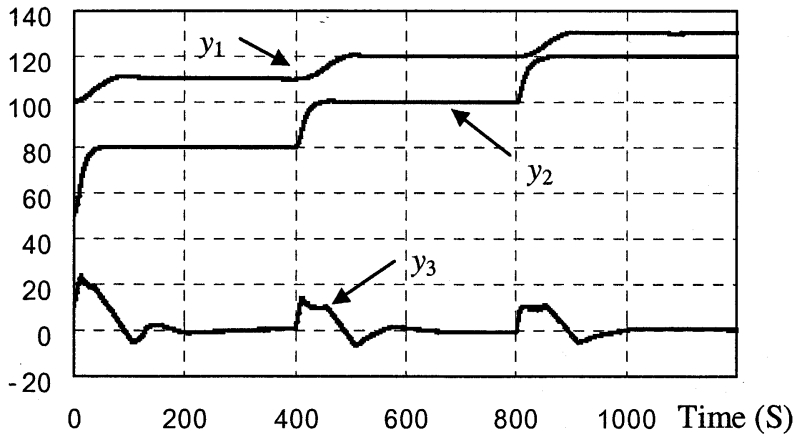

Fig. 13. Outputs of case 6.

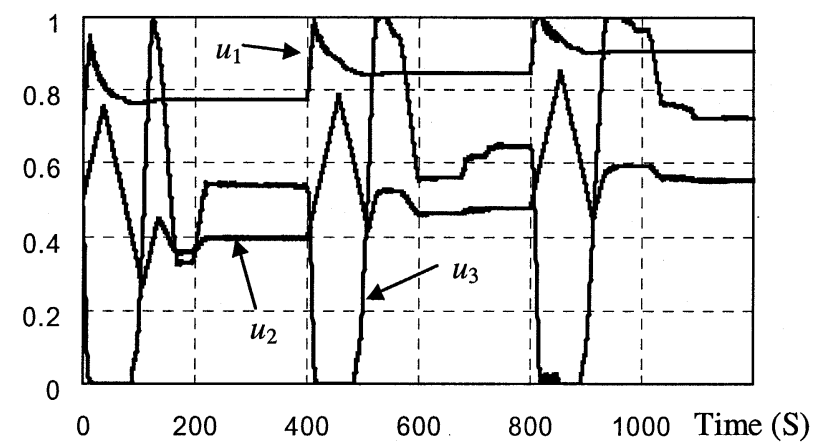

Fig. 14. Inputs of case 6 .

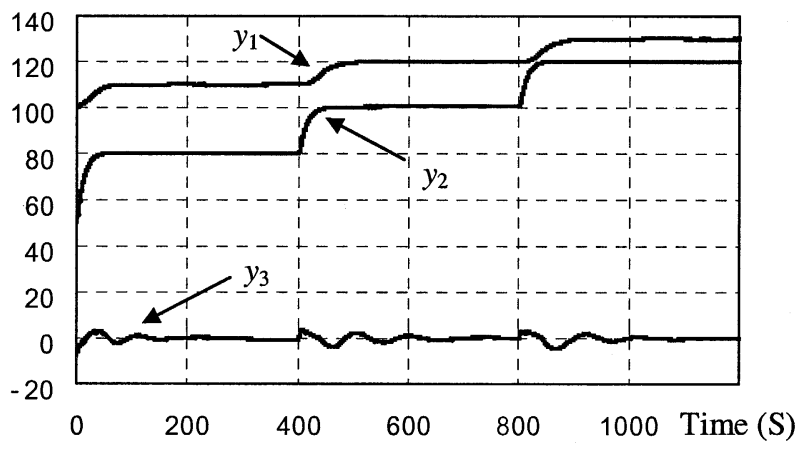

Fig. 15. Outputs of case 7.

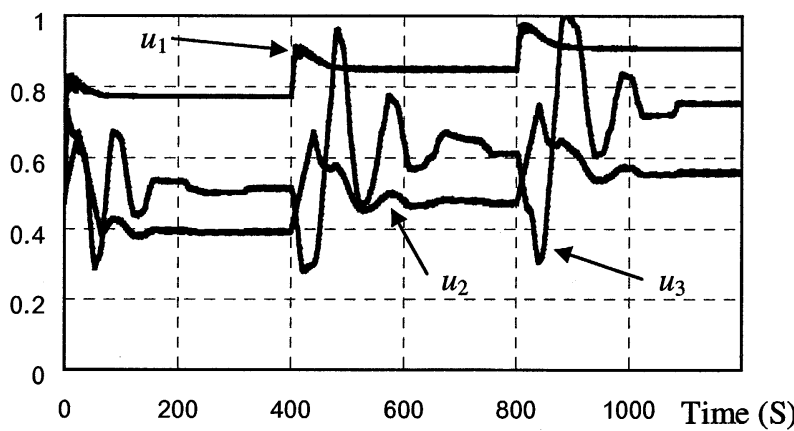

Fig. 16. Inputs of case 7.

that of case 4. This is because the effect of $a_{c s}$ on $y_{3}$ is reduced to $50 \%$ in (25), which makes smaller response of $y_{3}$ than that of case 4.

From cases 5, 6, and 7, the proposed controller shows satisfactory performances though the plant model is changed signif- icantly. This is because the proposed controller does not use the mathematical model of the system. Instead, the control rules are generated automatically with input-output history and the rule base is updated online to learn the behavior of the controlled plant.

The simulation results for various electric load demand changes and parameter variations show that the boiler-turbine system, which is highly complex and nonlinear, can be effectively controlled by the three single-loop FARMA FLCs.

\section{CONCLUSION}

This paper presents an application of online self-organizing fuzzy logic controller (SOFLC) to a boiler-turbine system in a fossil power plant. The control rules and the membership functions of FARMA FLC are generated automatically without using the plant model. The generated rules are stored in the fuzzy rule space and updated online by a self-organizing procedure. Three single loops are controlled with three FARMA FLCs. The boiler-turbine system considered is a highly nonlinear MIMO system. For various electric load demand changes and parameter variations, simulation results show that the MIMO nonlinear boiler-turbine system can be controlled effectively with the proposed SOFLC. Moreover, the proposed control system is shown to be adaptive and robust.

\section{REFERENCES}

[1] R. D. Bell and K. J. Åström, "Dynamic Models for Boiler-Turbine-Alternator Units: Data Logs and Parameter Estimation for a 160 MW Unit," Lund Institute of Technology, Sweden, Rep. TFRT-3192, 1987.

[2] B. W. Hogg and N. M. Ei-Rabaie, "Multivariable generalized predictive control of a boiler system," IEEE Trans. Energy Conversion, vol. 6, pp. 282-288, June 1991.

[3] J. A. Rovnak and R. Corlis, "Dynamic matrix based control of fossile power plant," IEEE Trans. Energy Conversion, vol. 6, pp. 320-326, June 1991.

[4] A. Ben-Abdennour and K. Y. Lee, "A decentralized controller design for a power plant using robust local controllers and functional mapping," IEEE Trans. Energy Conversion, vol. 11, pp. 394-400, June 1996.

[5] G. Prasad, E. Swidenbank, and B. W. Hogg, "A neural net model-based multivariable long-range predictive control strategy applied in thermal power plant control," IEEE Trans. Energy Conversion, vol. 13, pp. 176-182, Mar. 1991.

[6] R. Dimeo and K. Y. Lee, "Boiler-Turbine control system design using a genetic algorithm," IEEE Trans. Energy Conversion, vol. 10, pp. 752-759, Dec. 1995.

[7] F. A. Alturki and A. B. Abdennour, "Neuro-Fuzzy control of a Steam Boiler-Turbine unit," in Proc. IEEE Int. Conf. Control Applicat., Kohala Coast-Island of Hawaii, Hawaii, Aug. 1999, pp. 1050-1055.

[8] K. P. Cheung and L. X. Wang, "Comparision of Fuzzy and PI controllers for a Benchmark Drum-Boiler Model," in Proc. IEEE Int. Conf. Control Applicat., Trieste, Italy, 1998, pp. 958-962.

[9] Y. M. Park, U. C. Moon, and K. Y. Lee, "A Self-Organizing Fuzzy Logic Controller for dynamic systems using a Fuzzy Auto-Regressive Moving Average (FARMA) Model," IEEE Trans. Fuzzy Syst., vol. 3, pp. 75-82, Feb. 1995.

[10] — - "A Self-Organizing Power System Stabilizer using Fuzzy AutoRegressive Moving Average (FARMA) Model," IEEE Trans. Energy Conversion, vol. 11, pp. 442-448, June 1996.

[11] U. C. Moon, Y. M. Park, and K. Y. Lee, "A development of Power System Stabilizer with a Fuzzy Auto-Regressive Moving Average Model," Fuzzy Sets Syst., vol. 102, pp. 95-101, Feb. 1999.

[12] W. Pedrycz, Fuzzy Control and Fuzzy Systems. New York: Wiley, 1989. 


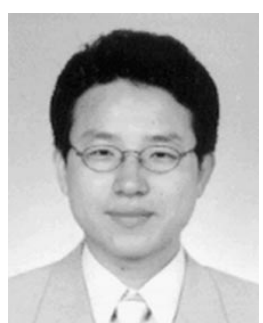

Un-Chul Moon received the B.S., M.S., and Ph.D. degrees in electrical engineering from Seoul National University, Seoul, Korea, in 1991, 1993, and 1996, respectively.

Currently, he is on the faculty of the School of Electrical Engineering at Chung-Ang University, Korea. He was with Samsung Company, Seoul, Korea, from 1996 to 2000, and then he was on the faculty of Electrical Engineering, Woosuk University, ChonBuk, Korea Institute of Control, from 2000 to 2002. His research interests include automatic control and intelligent system techniques and their application to power systems and industrial processes.

Dr. Moon received a "Young Researcher Paper Award 2001" from the Institute of Control, Automation and Systems Engineers, Seoul, Korea.

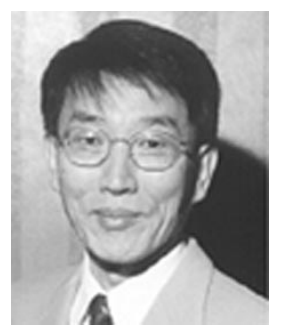

Kwang Y. Lee (F'01) received the B.S. degree in electrical engineering from Seoul National University, Seoul, Korea, in 1964, the M.Sc. degree in electrical engineering from North Dakota State University, Fargo, in 1968, and the Ph.D. degree in system science from Michigan State University, East Lansing, in 1971.

Currently, he is Professor of Electrical Engineering at Pennsylvania State University, University Park. He has been on the faculties of Michigan State, Oregon State, and University of Houston. His interests are power system control, operation and planning and intelligent system techniques, and their application to power system and power plant control.

Dr. Lee is an Associate Editor of IEEE TRANSACTIONS ON NEURAL NeTworks and Editor of IEEE TRANSACTIONS ON ENERGy CONVERSION. Dr. Lee is a Fellow of the IEEE. 